\title{
Sazonalidade da umidade de equilíbrio da madeira para o Estado de Mato Grosso
}

\author{
Adilson Pacheco de Souza ${ }^{1 *}$, Diego Martins Stangerlin¹, Rafael Rodolfo de Melo', Eduardo Morgan Uliana ${ }^{1}$ \\ ${ }^{1}$ Universidade Federal de Mato Grosso, Av. Alexandre Ferronatto 1200, St. Industrial, CEP 78557-267, Sinop, MG, Brasil
}

*Autor correspondente:

adilsonpacheco@ufmt.br

Termos para indexação:

Climatologia

Secagem natural

Higroscópico

Index terms:

Weather

Natural drying

Hygroscopicity

Histórico do artigo:

Recebido em 27/10/2014

Aprovado em 16/11/2016

Publicado em 30/12/2016

doi: $10.4336 / 2016 . p f b .36 .88 .811$

\begin{abstract}
Resumo - Por ser um material orgânico, heterogêneo e higroscópico, a madeira pode apresentar variações dimensionais e deformações decorrentes da interação com o ambiente. Em uso ou nos processos de secagem, quando são atingidos os equilíbrios entre a madeira e o ar atmosférico, têm-se a obtenção da umidade de equilíbrio da madeira (UEM). Em função da elevada importância dessa matéria-prima para o Estado de Mato Grosso, torna-se fundamental conhecer a sazonalidade de UEM. O presente trabalho objetivou estimar a UEM da madeira, pelo modelo de Simpson, para 30 municípios do Estado de Mato Grosso, distribuídos nas diferentes regiões de planejamento do Zoneamento Sócio-Econômico Ecológico do Estado. Os dados meteorológicos foram obtidos da Rede de Estações Meteorológicas Automáticas do Instituto Nacional de Meteorologia, entre 2006 e 2012. A UEM foi estimada diariamente, com posterior obtenção das médias mensais. Na estação seca ocorreram variações da UEM entre 5,08 e 9,57\% e no período chuvoso entre 8,58 e 13,49\%. Nos meses de janeiro, fevereiro e março ocorrem os maiores valores de UEM no estado. As condições ambientais durante o período seco (julho a setembro) favorecem a secagem natural da madeira serrada, por condicionarem uma secagem mais rápida.
\end{abstract}

\section{Seasonality of the wood moisture content equilibrium for Mato Grosso State, Brazil}

\begin{abstract}
Wood is an organic material, heterogeneous and hygroscopic which may present dimensional variation and deformation due to environment interaction. When moisture equilibrium between wood and atmospheric air is reached, in use or during drying, it is wood equilibrium moisture content (EMC). The wood presents high importance as raw material for Mato Grosso State. Them it becomes necessary to know the seasonality of the EMC. This study aimed to estimate the wood EMC by Simpson model for 30 cities in Mato Grosso State, Brazil, located in different regions of the socioeconomic and ecological zoning. Meteorological data from 2006 to 2012 were obtained from Automatic Weather Stations Network of the National Institute of Meteorology. EMC was estimated on daily databases to obtain monthly averages. EMC varied between 5.08 to $9.57 \%$ and $8.58 \%$ to $13.49 \%$ in the dry and rainy season, respectively. The higher EMC was observed from January to March. Wood dries naturally and faster due to environmental condition during dry season (June to September).
\end{abstract}




\section{Introdução}

A madeira possui uma série de propriedades que a torna uma matéria-prima de excelente qualidade, com baixa massa em relação ao volume, boa trabalhabilidade e boa adaptação como isolante térmico, elétrico e acústico. Além das várias qualidades, a possibilidade de escassez dos recursos naturais não-renováveis indica que, futuramente, a madeira possa ser considerada como a principal fonte de matéria-prima para a construção civil, fabricação de móveis e casas, dentre outros usos.

A madeira é um material orgânico e de estrutura complexa e heterogênea, com capacidade higroscópica variável e comportamento anisotrópico. Quando em uso, está sujeita a ocorrência de alterações dimensionais e deformações decorrentes das interações com a atmosfera (Kilic \& Niemz, 2012; Cassiano et al., 2013). Essas interações normalmente estão vinculadas a trocas gasosas, na forma de vapor d'água, e tendem a se estabilizar, até atingir o equilíbrio com a umidade do ar, definindo-se a umidade de equilíbrio da madeira (Kollmann \& Côté Junior, 1968; Skaar, 1972; Galvão, 1975; Jankowsky \& Galvão, 1979; Panshin \& De Zeeuw, 1980; Martins et al., 2003; Baraúna \& Oliveira, 2009). Este fenômeno pode ser entendido como o equilíbrio que ocorre quando a pressão interna de vapor d'água na parede celular tende a ser igual à pressão externa de vapor d'água (atmosfera).

Nesse sentido, a umidade da madeira, quando em equilíbrio com o ar atmosférico, é considerada como "umidade de equilíbrio da madeira" (UEM), sendo, portanto, um parâmetro dependente das variações sazonais da temperatura do $\operatorname{ar}(\mathrm{T})$ e da umidade relativa do ar (UR) da região. Esses elementos meteorológicos (T e UR) podem variar diariamente e sazonalmente, sendo que as variações diárias tendem a afetar a madeira apenas superficialmente (como rachaduras superficiais e oxidação de constituintes químicos), ao passo que, em longo prazo, podem causar alterações que comprometem os aspectos dimensionais e de qualidade da madeira, como empenamentos, inchamentos e contrações, dentre outros (Cassiano et al., 2013; Fioresi et al., 2014).

Além dos elementos meteorológicos, existem fatores intrínsecos da madeira que podem influenciar na UEM, como a estrutura anatômica e a composição química, que podem variar entre espécies, entre árvores da mesma espécie e na madeira de uma mesma árvore, com variações significativas nos sentidos base-topo (axial) e medula-casca (radial). Contudo, a T e a UR apresentam maior influência em função da elevada variabilidade espacial e temporal dessas variáveis (Galvão, 1975; Varejão-Silva, 2006).

Experimentalmente, a determinação da UEM pode ser baseada na exposição de amostras de madeira em ensaios de campo com ou sem exposição direta à radiação solar e precipitação pluviométrica (Pérez-Peña et al., 2011; Cassiano et al., 2013) ou em ensaios com câmaras climatizadas que apresentam condições controladas de T e UR (Baraúna \& Oliveira, 2009). Os ensaios em campo, apesar de demandarem mais tempo, propiciam resultados reais para o uso da madeira, visto que em laboratório a madeira não se sujeita às deteriorações causadas pelo intemperismo. Além dos ensaios em campo e laboratoriais, a UEM também pode ser obtida por meio de gráficos e/ou equações de estimativas, que consideram as variações de T e UR ligadas aos processos de absorção, adsorção e dessorção, independentemente da espécie (Baronasa et al., 2001; Mendes \& Arce, 2003; Chen et al., 2009).

Em geral, a aplicação das equações de estimativa de UEM consiste em uma referência interessante no processo de secagem da madeira, principalmente no controle do processo de secagem ao ar livre (Mendes $\&$ Arce, 2003). A maioria dos modelos de estimativa da UEM emprega a teoria de sorção da água na madeira proposta por Hailwood \& Harrobin (1946), considerando um ou dois hidratos (Martins et al., 2003; Baraúna \& Oliveira, 2009; Pérez-Peña et al., 2011).

O modelo de Simpson (1971) e/ou de Hailwood e Harrobin de um hidrato tem sido empregado na confecção das tabelas e/ou mapas de UEM disponíveis para muitas cidades do Brasil (Fioresi et al., 2014).

Contudo, ressalta-se a necessidade de avaliações em escala regional no Mato Grosso, visto que podem ser encontradas diferenças entre os valores de UEM convencionalmente adotados para o estado. Segundo Martins et al. (2003), pela aplicação do modelo de Simpson (1971), no estado do Mato Grosso, as oscilações de UEM ocorrem entre $14 \%$ e $16 \%$ nas médias anuais. Por sua vez, Cassiano et al. (2013) verificaram que esses níveis de UEM obtidas pelo mesmo modelo ocorrem apenas na estação chuvosa (outubro a abril) para a região de Sinop, MT. Em contrapartida, segundo a NBR 7190 (Associação Brasileira de Normas Técnicas, 1997), a condição-padrão para fins de utilização na construção civil é de UEM igual a 12\%, obtida para ambientes com UR igual ou superior a $65 \%$. 
Especificamente para madeiras tropicais, com diferentes massas específicas, na região Norte do Estado de Mato Grosso, Cassiano et al. (2013) concluíram que as estimativas de UEM pelos modelos de Simpson (1971) e/ou de Hailwood e Harrobin de um hidrato permitem melhores resultados para a obtenção de valores médios mensais para essa variável.

Nesse contexto, devido à crescente procura por madeira e por sua importância econômica para o Estado de Mato Grosso, e visando subsidiar programas de secagem ao ar livre nas regiões produtoras de madeira tropical, este trabalho objetivou estimar e avaliar a sazonalidade da umidade de equilíbrio da madeira, pelo modelo de Simpson (1971) para 30 municípios do estado, distribuídos nos biomas Amazônico, Cerrado e Pantanal e suas respectivas transições.

\section{Material e métodos}

Este estudo foi elaborado a partir de dados obtidos na rede de estações meteorológicas do Instituto de Nacional de Meteorologia (INMET), para 30 estações meteorológicas automáticas (EMA's) do Estado de Mato Grosso (Tabela 1). Segundo Souza et al. (2013), no Estado de Mato Grosso ocorrem dois tipos climáticos: Aw (clima tropical quente e úmido) e Cwa (clima temperado ou tropical de altitude), com temperaturas médias anuais variando de 23,00 a $26,84{ }^{\circ} \mathrm{C}$ e precipitações pluviais anuais oscilando entre 1.200 e $2.000 \mathrm{~mm}$, com maiores níveis nas regiões Norte e Médio Norte do Estado e nas regiões com altitudes próximas a $800 \mathrm{~m}$. Em geral, ocorrem concentrações da evapotranspiração potencial no verão inferiores a $40 \%$ e uma forte influência da latitude e da altitude nos padrões climáticos do Estado.

O Mato Grosso está localizado na região CentroOeste do Brasil, entre as coordenadas $06^{\circ} 00^{\prime} \mathrm{S}$ e $19^{\circ} 45^{\prime} \mathrm{S}$ e $50^{\circ} 06^{\prime} \mathrm{W}$ e $62^{\circ} 45^{\prime} \mathrm{W}$. O Estado apresenta área de $903.357,908 \mathrm{~km}^{2}$, representando $56,23 \%$ da Região Centro-Oeste e $10,61 \%$ de todo o território brasileiro.

Pelo Zoneamento Sócio-Econômico Ecológico do Estado (ZSEE-MT), que engloba aspectos do meio físico e biótico (geologia, geomorfologia, solos, clima, recursos hídricos, vegetação e fauna) e meio socioeconômico (dinâmica econômica, dinâmica demográfica e condições de vida da população) são compartimentadas 12 regiões de planejamento (Mato Grosso, 2014) (Tabela 1). A distribuição espacial das EMA's (Figura 1) foi implementada para obter a representatividade das diferentes regiões (categorias de uso) do ZSEE-MT.

Tabela 1. Estações meteorológicas automáticas do estado de Mato Grosso.

\begin{tabular}{|c|c|c|c|c|c|}
\hline Cidade & Região* & Latitude & Longitude & Altitude (m) & Período de dados \\
\hline Água Boa & IV & $-14,0161$ & $-52,2122$ & 432 & $01 / 2008-12 / 2012$ \\
\hline Alta Floresta & II & $-10,0672$ & $-56,7522$ & 294 & $09 / 2011-12 / 2012$ \\
\hline Apiacás & II & $-9,5639$ & $-57,3936$ & 220 & $10 / 2006-12 / 2012$ \\
\hline Campo Novo dos Parecis & VIII & $-13,7833$ & $-57,8333$ & 570 & $06 / 2010-12 / 2012$ \\
\hline Campo Verde & V & $-15,3139$ & $-55,0808$ & 749 & $01 / 2008-12 / 2012$ \\
\hline Carlinda & II & $-9,9703$ & $-55,8272$ & 300 & $04 / 2008-12 / 2012$ \\
\hline Comodoro & VII & $-13,4231$ & $-59,4546$ & 591 & $01 / 2008-12 / 2012$ \\
\hline Confresa & III & $-10,6539$ & $-51,5668$ & 237 & $06 / 2008-12 / 2012$ \\
\hline Cotriguaçu & I & $-9,9061$ & $-58,5719$ & 261 & $01 / 2008-01 / 2013$ \\
\hline Cuiabá & VI & $-15,5594$ & $-56,0628$ & 240 & 05/2011 - 12/2012 \\
\hline Gaucha do Norte & IV & $-13,1847$ & $-53,2575$ & 379 & $08 / 2008-12 / 2012$ \\
\hline Guarantã & XII & $-9,9500$ & $-54,8833$ & 320 & $05 / 2007-12 / 2012$ \\
\hline Guiratinga & VI & $-16,3417$ & $-53,7661$ & 526 & $01 / 2008-12 / 2012$ \\
\hline Itiquira & VI & $-17,1750$ & $-54,5014$ & 585 & $08 / 2008-12 / 2012$ \\
\hline Juara & XI & $-11,2803$ & $-57,5267$ & 260 & $11 / 2006-12 / 2012$ \\
\hline Juína & I & $-11,3750$ & $-58,7750$ & 374 & $10 / 2007-12 / 2012$ \\
\hline
\end{tabular}

Continua... 
Tabela 1. Continuação.

\begin{tabular}{lccccc}
\hline \multicolumn{1}{c}{ Cidade } & Região* & Latitude & Longitude & Altitude (m) & Período de dados \\
& & & & & \\
\hline Nova Maringá & XI & $-13,0386$ & $-57,0922$ & 353 & $04 / 2008-12 / 2012$ \\
Nova Ubiratã & X & $-13,4111$ & $-54,7522$ & 518 & $04 / 2008-12 / 2012$ \\
Novo Mundo & VIII & $-12,5219$ & $-58,2314$ & 431 & $03 / 2008-12 / 2012$ \\
Pontes de Lacerda & VII & $-15,2511$ & $-59,3467$ & 256 & $01 / 2008-12 / 2012$ \\
Porto Estrela & VII & $-15,3247$ & $-57,2264$ & 145 & $02 / 2008-12 / 2012$ \\
Rondonópolis & VI & $-16,4500$ & $-54,5666$ & 284 & $01 / 2008-12 / 2012$ \\
Salto do Céu & VII & $-15,1247$ & $-58,1275$ & 303 & $01 / 2008-12 / 2012$ \\
Santo Antonio do Leste & III & $-14,9278$ & $-53,8836$ & 648 & $08 / 2008-12 / 2012$ \\
São Félix do Araguaia & III & $-11,6189$ & $-50,7278$ & 218 & $08 / 2011-12 / 2012$ \\
São José do Rio Claro & IX & $-13,4500$ & $-56,6666$ & 350 & $03 / 2010-12 / 2012$ \\
Sinop & XII & $-11,9822$ & $-55,5658$ & 371 & $11 / 2006-12 / 2012$ \\
Sorriso & X & $-12,5452$ & $-55,7113$ & 380 & $01 / 2009-12 / 2012$ \\
Tangará da Serra & VIII & $-14,6500$ & $-57,4315$ & 321 & $01 / 2008-12 / 2012$ \\
Vila Bela S. Trindade & VII & $-15,0628$ & $-59,8729$ & 222 & $01 / 2008-12 / 2012$ \\
\hline
\end{tabular}

*Regiões de planejamento do Estado de Mato Grosso: I - Noroeste 1; II - Norte; III - Nordeste; IV - Leste; V - Sudeste; VI - Sul; VII - Sudoeste; VIII - Oeste; IX - Centro Oeste; X - Centro; XI - Noroeste 2; XII - Centro Norte. Fonte: Mato Grosso (2014)

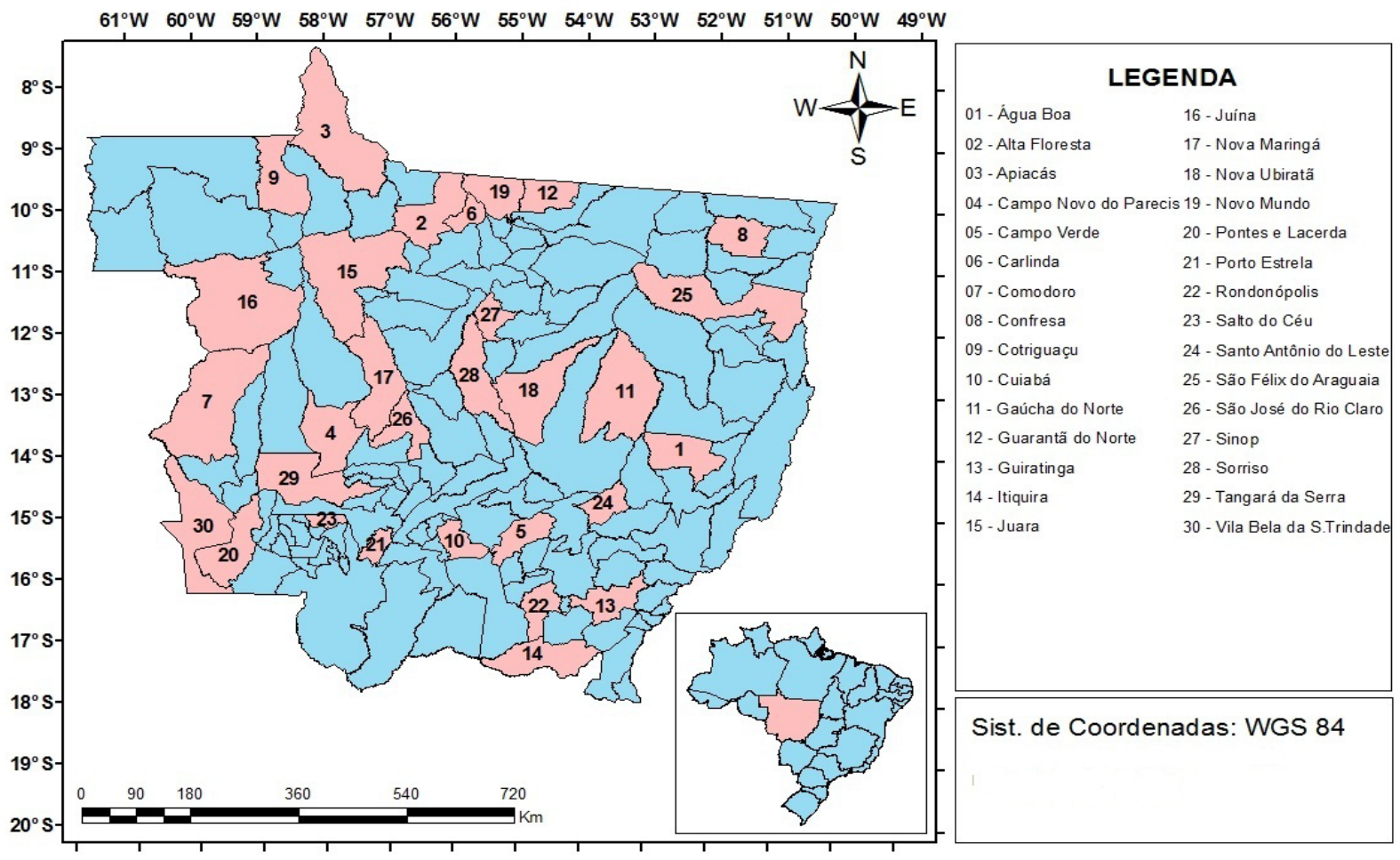

Figura 1. Distribuição das estações meteorológicas automáticas avaliadas no Estado de Mato Grosso. 
A umidade de equilíbrio da madeira (UEM) foi estimada pelo método de Simpson (1971), equação 1, conforme recomendações de Cassiano et al. (2013). Esse método baseia-se na teoria da adsorção de Hailwood e Horrobin e permite obter UEM em função da temperatura (T) e da umidade relativa (UR) (equações 2, 3 e 4).

$$
\begin{aligned}
& \mathrm{UEM}=\left[\frac{\mathrm{K}_{1} \mathrm{~K}_{2} \mathrm{H}}{1+\mathrm{K}_{1} \mathrm{~K}_{2} \mathrm{H}}+\frac{\mathrm{K}_{2} \mathrm{H}}{1-\mathrm{K}_{2} \mathrm{H}}\right] \cdot \frac{1800}{\mathrm{~W}} \\
& \mathrm{~K} 1=4,737+0,04773 \mathrm{~T}_{\mathrm{C}}-0,00050123 \mathrm{~T}_{\mathrm{C}}{ }^{2} \\
& \mathrm{~K} 2=0,705941+0,001698 \mathrm{Tc}-0,000005553 \mathrm{Tc}^{2} \\
& \mathrm{~W}=223,384+06942 \mathrm{Tc}+0,0185324 \mathrm{Tc}^{2}
\end{aligned}
$$

em que: UEM = umidade de equilíbrio da madeira $(\%) ; \mathrm{H}=$ pressão de vapor relativa (UR $\left.100^{-1}\right) ; \mathrm{W}=$ peso molecular da unidade polimérica que forma o hidrato; K1 e K2 são constantes de equilíbrio, obtidas em função da temperatura diária $\left(\mathrm{Tc}, \mathrm{em}^{\circ} \mathrm{C}\right)$.

A distribuição espacial dos valores médios de UEM para o estado de Mato Grosso foi obtida por meio de interpolação por krigagem ordinária, para as estações hídricas (seca e chuvosa) pelo emprego do software ArcGis.

\section{Resultados e discussão}

De modo geral, em algumas regiões do Mato Grosso existe uma tendência de alteração da sazonalidade das médias mensais das temperaturas $(\mathrm{T})$ quando comparadas com outras regiões brasileiras, visto que as maiores médias mensais de $\mathrm{T}$ normalmente ocorrem entre agosto e outubro (Tabela 2). Contudo, para séries temporais inferiores às normais climatológicas (30 anos) não se recomenda atribuir possíveis causas (El Nino/Oscilação Sul; aquecimento global; alteração climática cíclica; ações antrópicas, dentre outras) para as tendências observadas. Por conseguinte, a identificação desses ciclos é fundamental para nortear estratégias de adaptação e avaliar os seus efeitos em outras variáveis ambientais, como a umidade de equilíbrio da madeira (UEM) (Streck et al., 2011).

A umidade relativa (UR) apresenta tendência de evolução diurna inversa à T (Varejão-Silva, 2006). Esse comportamento decorre da dependência exponencial da
UR em relação a $T$, visto que a sua obtenção usualmente é estabelecida pela equação de Tétens, sendo que o aumento ou redução de UR não significa a ocorrência de mudanças na concentração de vapor d'água do ar, pois com o aumento de $\mathrm{T}$, ocorre a expansão da massa de ar e aumenta a capacidade da mesma em conter vapor d'água (pressão de saturação - es), contudo, a UR pode diminuir em razão da pequena variação da pressão parcial de vapor d'água (ea) e vice-versa. Como consequência desse efeito, espera-se que a UR apresente valores mínimos nos meses de maior $T$ e máximos nos meses com menor $\mathrm{T}$, corroborando com os dados médios mensais da Tabela 3.

Além disso, notam-se diferenças entre os valores médios mensais de UR nas estações chuvosa e seca, visto que essa variável pode ser considerada como um indicador da formação e/ou presença de vapor d'água na atmosfera, nuvens e, consequentemente, das precipitações pluviais. Esse comportamento corrobora com as observações em outras regiões brasileiras e no próprio Estado de Mato Grosso (Alves \& Biudes, 2012; Queiroz \& Costa, 2012; Medeiros et al., 2013; Santos et al., 2013). As menores médias de UR ocorrem nas regiões Nordeste, Leste e Sudeste, que correspondem às regiões das bacias hidrográficas do AraguaiaTocantins e Paraguai, caracterizadas como as regiões com os menores níveis pluviométricos do Estado (Souza et al., 2013). Em geral, as médias mensais de UR variaram entre 39,63\% (Santo Antônio do Leste) e $89,50 \%$ (Cotriguaçu), nos meses de agosto e janeiro, respectivamente. Nesse sentido, considerando as médias para o Estado (média de todos os municípios), têm-se 49,35 e $82,73 \%$ para os meses de agosto e janeiro e, na média anual em torno de $71,84 \%$.

Por conseguinte, pelo controle da UR é possível obter informações para avaliação das taxas de saída de umidade e controle das tensões que se desenvolvem na madeira devido às variações dimensionais que ocorrem durante a secagem (Silva \& Oliveira, 2003). Em condições de secagem natural, a madeira perderá água na forma de vapor até atingir a UE da região. Em regiões secas e quentes, a madeira apresentará UE menor, quando comparada com regiões frias e úmidas. Nesse contexto, as maiores e menores médias mensais de UEM estimadas pelo método de Simpson (1971) ocorreram na região Noroeste do estado, para Cotriguaçu $(15,21 \pm 1,78 \%)$ e Juara $(3,56 \pm 2,94 \%)$, em janeiro e agosto, respectivamente (Tabela 4). 
Tabela 2. Médias mensais da temperatura do $\operatorname{ar}\left(\mathrm{em}^{\circ} \mathrm{C}\right)$ para os diferentes municípios do Estado de Mato Grosso.

\begin{tabular}{|c|c|c|c|c|c|c|c|c|c|c|c|c|c|}
\hline \multirow{2}{*}{ Cidades } & \multicolumn{13}{|c|}{ Período } \\
\hline & Jan & Fev & Mar & Abr & Mai & Jun & Jul & Ago & Set & Out & Nov & Dez & Anual \\
\hline Água Boa & 24,59 & 24,60 & 24,97 & 25,10 & 24,28 & 24,28 & 24,28 & 25,99 & 27,89 & 26,77 & 25,03 & 24,76 & 25,21 \\
\hline Alta Floresta & - & - & 24,54 & 25,10 & 24,55 & 23,70 & 23,19 & 24,43 & 26,86 & 25,47 & 24,91 & 24,98 & 24,77 \\
\hline Apiacás & 25,42 & 24,81 & 24,51 & 25,38 & 25,58 & 25,61 & 25,91 & 26,84 & 26,93 & 26,33 & 25,51 & 25,89 & 25,73 \\
\hline Campo Novo dos Parecis & 24,74 & 25,89 & 28,55 & 26,67 & 23,40 & 23,23 & 22,71 & 24,89 & 27,35 & 26,05 & 26,02 & 26,25 & 25,48 \\
\hline Campo Verde & 22,89 & 23,19 & 23,37 & 23,09 & 21,56 & 21,56 & 22,19 & 23,96 & 25,49 & 24,44 & 23,66 & 23,21 & 23,22 \\
\hline Carlinda & 25,26 & 25,46 & 25,31 & 25,58 & 25,47 & 25,41 & 25,96 & 27,08 & 26,92 & 25,88 & 25,45 & 25,13 & 25,74 \\
\hline Comodoro & 23,17 & 22,99 & 23,11 & 22,79 & 21,48 & 20,77 & 20,80 & 21,65 & 23,48 & 23,84 & 23,59 & 23,30 & 22,58 \\
\hline Confresa & 25,35 & 25,58 & 26,20 & 26,29 & 26,48 & 26,12 & 26,33 & 27,75 & 28,98 & 27,22 & 26,09 & 25,90 & 26,53 \\
\hline Cotriguaçu & 24,44 & 24,77 & 24,61 & 25,02 & 24,63 & 24,14 & 24,21 & 25,30 & 26,10 & 25,84 & 25,25 & 24,90 & 24,93 \\
\hline Cuiabá & 26,21 & 25,93 & 26,18 & 26,64 & 23,19 & 22,47 & 22,70 & 26,26 & 29,56 & 28,32 & 27,37 & 27,13 & 26,00 \\
\hline Gaucha do Norte & 24,88 & 25,38 & 25,79 & 25,98 & 25,36 & 24,90 & 25,05 & 25,65 & 27,33 & 26,73 & 25,38 & 25,08 & 25,63 \\
\hline Guarantã & 24,81 & 25,26 & 25,34 & 26,16 & 25,38 & 24,81 & 24,99 & 25,58 & 26,69 & 26,04 & 25,49 & 25,19 & 25,48 \\
\hline Guiratinga & 24,89 & 24,77 & 25,14 & 25,01 & 23,58 & 23,37 & 23,26 & 25,28 & 27,33 & 26,96 & 25,55 & 25,35 & 25,04 \\
\hline Itiquira & 24,36 & 24,68 & 24,80 & 24,24 & 23,08 & 22,74 & 22,40 & 24,68 & 26,44 & 25,74 & 25,11 & 24,88 & 24,43 \\
\hline Juara & 25,70 & 25,32 & 25,61 & 25,75 & 25,06 & 24,61 & 25,48 & 27,58 & 27,68 & 27,32 & 26,45 & 25,72 & 26,02 \\
\hline Juína & 24,77 & 24,66 & 24,62 & 24,62 & 24,71 & 24,16 & 24,26 & 25,67 & 26,76 & 25,99 & 25,19 & 25,06 & 25,04 \\
\hline Nova Maringá & 26,93 & 27,75 & 27,60 & 26,13 & 24,75 & 23,49 & 23,50 & 25,98 & 26,41 & 26,98 & 26,09 & 25,61 & 25,94 \\
\hline Nova Ubiratã & 23,73 & 24,90 & 25,15 & 25,07 & 23,98 & 23,76 & 24,27 & 26,13 & 27,35 & 26,10 & 24,78 & 24,36 & 24,97 \\
\hline Novo Mundo & 24,09 & 24,36 & 24,40 & 24,64 & 24,09 & 23,98 & 24,67 & 26,73 & 27,72 & 26,18 & 24,97 & 24,40 & 25,02 \\
\hline Pontes e Lacerda & 25,88 & 25,52 & 25,77 & 25,09 & 23,44 & 22,77 & 23,44 & 25,40 & 27,05 & 27,55 & 26,36 & 25,97 & 25,35 \\
\hline Porto Estrela & 26,55 & 26,12 & 26,16 & 26,36 & 25,12 & 23,17 & 23,01 & 24,48 & 27,50 & 27,84 & 27,33 & 26,75 & 25,87 \\
\hline Rondonópolis & 25,35 & 25,46 & 25,43 & 25,05 & 23,20 & 22,39 & 22,64 & 24,95 & 28,01 & 27,84 & 26,45 & 26,23 & 25,25 \\
\hline Salto do Céu & 25,37 & 25,29 & 25,18 & 24,45 & 22,61 & 21,86 & 21,88 & 23,60 & 25,84 & 26,39 & 25,97 & 25,54 & 24,50 \\
\hline Santo Antonio do Leste & 23,67 & 24,05 & 24,35 & 23,80 & 23,78 & 23,01 & 23,06 & 25,18 & 26,34 & 25,21 & 24,07 & 23,63 & 24,18 \\
\hline São Félix do Araguaia & 25,21 & 25,73 & 25,93 & 27,05 & 24,66 & 22,56 & 23,11 & 27,86 & 28,86 & 27,30 & 24,58 & 25,11 & 25,66 \\
\hline São José do Rio Claro & - & - & 25,74 & 25,53 & 23,63 & 23,46 & 21,83 & 23,35 & 27,82 & - & - & - & 24,48 \\
\hline Sinop & 24,52 & 24,37 & 25,15 & 25,00 & 24,41 & 24,42 & 25,06 & 26,64 & 27,00 & 25,92 & 25,37 & 24,43 & 25,19 \\
\hline Sorriso & 24,98 & 25,09 & 25,65 & 25,85 & 25,15 & 24,81 & 25,52 & 26,69 & 28,42 & 27,02 & 25,49 & 25,61 & 25,86 \\
\hline Tangará da Serra & 24,39 & 24,17 & 24,37 & 24,91 & 23,50 & 23,00 & 22,83 & 25,65 & 27,11 & 26,12 & 25,18 & 25,32 & 24,71 \\
\hline Vila Bela S. Trindade & 25,78 & 25,66 & 25,76 & 25,23 & 23,69 & 23,05 & 23,33 & 24,56 & 26,71 & 27,12 & 26,54 & 26,23 & 25,31 \\
\hline
\end{tabular}


Tabela 3. Médias mensais da umidade relativa do ar (em \%) para os diferentes municípios do Estado de Mato Grosso.

\begin{tabular}{|c|c|c|c|c|c|c|c|c|c|c|c|c|c|}
\hline \multirow{2}{*}{ Cidades } & \multicolumn{13}{|c|}{ Período } \\
\hline & Jan & Fev & Mar & Abr & Mai & Jun & Jul & Ago & Set & Out & Nov & Dez & Anual \\
\hline Água Boa & 82,91 & 82,49 & 82,00 & 78,60 & 69,50 & 64,63 & 54,13 & 39,99 & 44,64 & 66,69 & 80,37 & 82,23 & 69,01 \\
\hline Alta Floresta & - & - & 85,99 & 85,52 & 82,14 & 81,12 & 70,49 & 58,63 & 54,71 & 77,51 & 84,41 & 84,89 & 76,54 \\
\hline Apiacás & 84,79 & 82,30 & 84,09 & 81,50 & 78,56 & 73,53 & 64,61 & 59,65 & 62,33 & 73,52 & 82,72 & 83,57 & 75,93 \\
\hline Campo Novo dos Parecis & 80,35 & 74,55 & 65,66 & 78,08 & 74,98 & 65,97 & 59,08 & 44,98 & 49,95 & 67,42 & 71,03 & 71,47 & 66,96 \\
\hline Campo Verde & 83,70 & 82,80 & 82,31 & 79,75 & 75,05 & 67,43 & 53,44 & 43,72 & 50,25 & 70,27 & 79,47 & 82,50 & 70,89 \\
\hline Carlinda & 82,66 & 81,56 & 83,18 & 81,20 & 76,19 & 66,44 & 54,22 & 47,70 & 65,24 & 76,94 & 81,37 & 82,65 & 73,28 \\
\hline Comodoro & 87,74 & 87,61 & 86,91 & 85,32 & 82,12 & 78,64 & 70,80 & 67,87 & 72,79 & 79,42 & 84,89 & 85,52 & 80,80 \\
\hline Confresa & 82,67 & 81,88 & 82,11 & 79,86 & 72,96 & 63,19 & 52,29 & 42,13 & 49,06 & 69,48 & 78,51 & 78,93 & 69,42 \\
\hline Cotriguaçu & 89,50 & 87,23 & 87,86 & 84,73 & 80,97 & 74,44 & 64,56 & 58,11 & 73,84 & 79,85 & 85,13 & 86,27 & 79,38 \\
\hline Cuiabá & 76,40 & 76,53 & 75,46 & 75,10 & 77,70 & 73,17 & 64,90 & 43,23 & 42,73 & 61,29 & 70,13 & 72,42 & 67,42 \\
\hline Gaucha do Norte & 81,91 & 79,85 & 79,24 & 75,02 & 68,35 & 62,00 & 54,32 & 46,83 & 51,72 & 69,30 & 78,96 & 80,62 & 69,01 \\
\hline Guarantã & 86,52 & 83,11 & 82,43 & 79,22 & 78,30 & 72,94 & 65,18 & 59,23 & 68,11 & 78,81 & 83,42 & 84,78 & 76,84 \\
\hline Guiratinga & 79,01 & 81,57 & 80,76 & 76,61 & 70,02 & 62,47 & 51,77 & 42,23 & 46,84 & 62,92 & 76,39 & 78,02 & 67,38 \\
\hline Itiquira & 79,85 & 81,34 & 80,00 & 72,05 & 69,42 & 61,75 & 54,86 & 41,32 & 47,57 & 66,12 & 75,89 & 79,58 & 67,48 \\
\hline Juara & 79,85 & 83,62 & 84,04 & 82,87 & 78,35 & 72,31 & 57,70 & 49,45 & 63,16 & 71,26 & 65,14 & 66,56 & 71,19 \\
\hline Juína & 85,77 & 86,09 & 85,65 & 85,65 & 78,09 & 72,24 & 64,18 & 53,76 & 65,68 & 76,79 & 82,03 & 82,79 & 76,56 \\
\hline Nova Maringá & 82,93 & 82,00 & 81,86 & 78,98 & 73,47 & 70,68 & 61,85 & 51,22 & 65,50 & 69,88 & 79,17 & 80,97 & 73,21 \\
\hline Nova Ubiratã & 86,83 & 80,87 & 80,49 & 80,50 & 70,71 & 59,49 & 50,13 & 41,48 & 49,15 & 70,12 & 80,89 & 83,04 & 69,47 \\
\hline Novo Mundo & 87,39 & 84,54 & 85,22 & 81,74 & 74,81 & 66,71 & 54,06 & 45,51 & 54,45 & 71,44 & 82,88 & 85,41 & 72,85 \\
\hline Pontes e Lacerda & 79,14 & 83,03 & 82,39 & 77,90 & 74,67 & 70,01 & 60,18 & 52,89 & 54,64 & 62,96 & 74,40 & 76,58 & 70,73 \\
\hline Porto Estrela & 79,05 & 85,02 & 84,88 & 82,07 & 78,70 & 74,72 & 67,53 & 57,06 & 56,76 & 66,46 & 73,52 & 77,11 & 73,57 \\
\hline Rondonópolis & 78,68 & 79,03 & 78,98 & 77,81 & 75,15 & 70,38 & 60,01 & 47,69 & 47,74 & 64,70 & 73,55 & 76,50 & 69,18 \\
\hline Salto do Céu & 81,89 & 83,14 & 83,85 & 81,97 & 80,45 & 76,00 & 66,98 & 58,63 & 60,06 & 69,81 & 76,88 & 79,81 & 74,96 \\
\hline Santo Antonio do Leste & 81,59 & 80,23 & 79,69 & 67,63 & 62,08 & 62,60 & 59,67 & 39,63 & 47,41 & 68,60 & 78,91 & 82,10 & 67,51 \\
\hline São Félix do Araguaia & 83,31 & 80,06 & 81,81 & 77,93 & 78,93 & 76,98 & 64,68 & 39,71 & 44,06 & 69,12 & 82,14 & 79,70 & 71,54 \\
\hline São José do Rio Claro & - & - & 83,05 & 76,45 & 72,82 & 69,76 & 64,35 & 54,77 & 45,22 & - & - & - & 66,63 \\
\hline Sinop & 86,14 & 86,02 & 81,97 & 82,59 & 75,15 & 62,91 & 52,91 & 45,91 & 56,43 & 74,11 & 80,06 & 85,97 & 72,51 \\
\hline Sorriso & 80,41 & 77,73 & 77,29 & 77,01 & 72,99 & 64,42 & 52,72 & 46,38 & 50,61 & 67,81 & 77,69 & 77,00 & 68,50 \\
\hline Tangará da Serra & 83,99 & 82,18 & 81,63 & 80,66 & 77,52 & 73,00 & 61,07 & 41,77 & 49,87 & 71,41 & 80,57 & 80,59 & 72,02 \\
\hline Vila Bela S. Trindade & 81,55 & 83,92 & 84,48 & 81,96 & 78,66 & 74,32 & 65,95 & 58,99 & 59,83 & 68,07 & 76,94 & 79,24 & 74,49 \\
\hline
\end{tabular}

- Dados não disponíveis 
Tabela 4. Médias mensais da umidade de equilíbrio da madeira (em \%) para os diferentes municípios do Estado de Mato Grosso.

\begin{tabular}{|c|c|c|c|c|c|c|c|c|c|c|c|c|c|c|c|}
\hline \multirow{2}{*}{ Cidades } & \multicolumn{12}{|c|}{ Meses } & \multicolumn{3}{|c|}{ Anual } \\
\hline & Jan & Fev & Mar & Abr & Mai & Jun & Jul & Ago & Set & Out & Nov & Dez & Mínima & Máxima & Média \\
\hline Água Boa & 12,55 & 12,41 & 12,19 & 11,16 & 8,72 & 6,93 & 4,90 & 3,80 & 9,92 & 8,33 & 11,65 & 12,31 & 3,80 & 12,55 & 9,57 \\
\hline Alta Floresta & - & - & 13,57 & 13,36 & 12,11 & 11,81 & 8,75 & 6,31 & 6,48 & 10,87 & 12,99 & 13,15 & 6,31 & 13,57 & 10,94 \\
\hline Apiacás & 13,24 & 12,27 & 12,91 & 11,95 & 11,02 & 9,49 & 7,38 & 6,45 & 7,30 & 9,80 & 12,45 & 12,67 & 6,45 & 13,24 & 10,58 \\
\hline Campo Novo dos Parecis & 11,58 & 9,85 & 7,56 & 10,79 & 10,07 & 7,90 & 6,45 & 4,38 & 5,08 & 8,19 & 9,10 & 9,09 & 4,38 & 11,58 & 8,34 \\
\hline Campo Verde & 13,02 & 12,52 & 12,36 & 11,64 & 10,39 & 8,66 & 5,95 & 4,49 & 5,57 & 9,03 & 11,44 & 12,47 & 4,49 & 13,02 & 9,80 \\
\hline Carlinda & 12,45 & 12,07 & 12,54 & 11,92 & 10,38 & 7,97 & 5,60 & 4,76 & 7,83 & 10,54 & 11,95 & 12,42 & 4,76 & 12,54 & 10,04 \\
\hline Comodoro & 14,36 & 14,35 & 14,12 & 13,49 & 12,44 & 11,25 & 9,20 & 8,35 & 9,46 & 11,41 & 13,23 & 13,52 & 8,35 & 14,36 & 12,10 \\
\hline Confresa & 12,39 & 12,07 & 12,12 & 11,55 & 9,48 & 7,19 & 5,28 & 3,97 & 5,14 & 8,75 & 10,99 & 11,10 & 3,97 & 12,39 & 9,17 \\
\hline Cotriguaçu & 15,21 & 14,27 & 14,54 & 13,41 & 12,02 & 10,06 & 7,63 & 6,52 & 9,66 & 11,41 & 13,27 & 13,80 & 6,52 & 15,21 & 11,82 \\
\hline Cuiabá & 10,31 & 10,35 & 10,03 & 9,93 & 11,19 & 10,12 & 8,37 & 4,49 & 4,24 & 6,87 & 8,90 & 9,26 & 4,24 & 11,19 & 8,67 \\
\hline Gaucha do Norte & 12,14 & 11,45 & 11,25 & 10,08 & 8,38 & 6,99 & 5,60 & 4,61 & 5,48 & 8,70 & 11,13 & 11,75 & 4,61 & 12,14 & 8,96 \\
\hline Guarantã & 13,85 & 12,53 & 12,23 & 11,24 & 10,97 & 9,40 & 7,54 & 6,46 & 8,34 & 11,09 & 12,63 & 13,15 & 6,46 & 13,85 & 10,79 \\
\hline Guiratinga & 11,47 & 12,08 & 11,87 & 10,75 & 8,88 & 7,27 & 5,45 & 4,11 & 5,00 & 7,40 & 10,46 & 11,00 & 4,11 & 12,08 & 8,81 \\
\hline Itiquira & 11,81 & 12,04 & 11,81 & 9,64 & 8,89 & 7,47 & 6,47 & 4,22 & 5,19 & 8,14 & 10,37 & 11,58 & 4,22 & 12,04 & 8,97 \\
\hline Juara & 8,31 & 8,52 & 8,54 & 8,31 & 7,32 & 6,24 & 4,19 & 3,56 & 7,37 & 9,04 & 8,91 & 9,72 & 3,56 & 9,72 & 7,50 \\
\hline Juína & 13,52 & 13,68 & 13,46 & 12,53 & 10,90 & 9,47 & 7,54 & 5,67 & 7,84 & 10,48 & 12,13 & 12,46 & 5,67 & 13,68 & 10,81 \\
\hline Nova Maringá & 12,47 & 12,07 & 11,99 & 11,11 & 9,58 & 8,92 & 6,97 & 5,32 & 7,78 & 8,70 & 11,19 & 11,82 & 5,32 & 12,47 & 9,83 \\
\hline Nova Ubiratã & 14,01 & 11,80 & 11,65 & 11,66 & 8,99 & 6,19 & 5,04 & 3,91 & 5,17 & 8,94 & 11,80 & 12,59 & 3,91 & 14,01 & 9,31 \\
\hline Novo Mundo & 14,29 & 13,14 & 13,41 & 12,20 & 10,05 & 8,10 & 5,65 & 4,48 & 6,02 & 9,14 & 12,67 & 13,50 & 4,48 & 14,29 & 10,22 \\
\hline Pontes de Lacerda & 11,29 & 12,52 & 12,26 & 10,87 & 10,00 & 8,76 & 6,75 & 5,55 & 5,77 & 7,20 & 9,91 & 10,55 & 5,55 & 12,52 & 9,29 \\
\hline Porto Estrela & 11,22 & 13,22 & 13,12 & 12,10 & 11,08 & 9,97 & 8,16 & 6,14 & 6,09 & 7,97 & 9,58 & 10,63 & 6,09 & 13,22 & 9,94 \\
\hline Rondonopolis & 11,19 & 11,07 & 11,11 & 10,77 & 10,07 & 8,91 & 6,79 & 4,80 & 4,93 & 7,68 & 9,63 & 10,38 & 4,80 & 11,19 & 8,94 \\
\hline Salto do Céu & 12,13 & 12,55 & 12,80 & 12,13 & 11,66 & 10,36 & 8,17 & 6,53 & 6,70 & 8,71 & 10,54 & 11,41 & 6,53 & 12,80 & 10,31 \\
\hline Santo Antonio do Leste & 12,20 & 11,48 & 11,64 & 9,00 & 7,56 & 7,34 & 7,21 & 3,60 & 5,07 & 8,65 & 11,19 & 12,25 & 3,60 & 12,25 & 8,93 \\
\hline São Félix do Araguaia & 12,61 & 11,55 & 12,07 & 10,73 & 11,21 & 10,52 & 7,88 & 3,64 & 4,20 & 8,65 & 12,17 & 11,43 & 3,64 & 12,61 & 9,72 \\
\hline São José do Rio Claro & - & - & 12,49 & 10,35 & 9,39 & 8,59 & 7,46 & 5,67 & 4,27 & - & - & - & 4,27 & 12,49 & 8,32 \\
\hline Sinop & 13,71 & 13,67 & 12,25 & 12,45 & 10,12 & 7,29 & 5,48 & 4,60 & 6,38 & 9,84 & 11,55 & 13,63 & 4,60 & 13,71 & 10,08 \\
\hline Sorriso & 11,61 & 10,78 & 10,58 & 10,46 & 9,59 & 7,58 & 5,38 & 4,55 & 5,21 & 8,19 & 10,69 & 10,59 & 4,55 & 11,61 & 8,77 \\
\hline Tangará da Serra & 12,89 & 12,22 & 11,99 & 11,67 & 10,83 & 9,86 & 7,10 & 3,99 & 5,40 & 9,05 & 11,77 & 11,65 & 3,99 & 12,89 & 9,87 \\
\hline Vila Bela S. Trindade & 12,12 & 12,84 & 13,01 & 12,09 & 11,09 & 9,96 & 7,97 & 6,54 & 6,68 & 8,33 & 10,57 & 11,35 & 6,54 & 13,01 & 10,21 \\
\hline
\end{tabular}

- Dados não disponíveis. 
Em geral, corroborando com a tendência da UR, os valores mensais mínimos da UEM ocorrem em julho e/ou agosto para $90 \%$ dos municípios, ou ainda, com postergação para setembro (Cuiabá, Porto Estrela e São José do Rio Claro), contudo, ainda no período seco. Quanto aos valores mensais máximos da UEM, ocorrem variações entre os meses de janeiro, fevereiro e março, sem observações de tendências de distribuição no estado. As variações anuais da UEM nas diferentes regiões indicam amplitudes mensais entre $6,01 \%$ (Comodoro) e $10,1 \%$ (Nova Ubiratã), sendo que as regiões Sudoeste e Oeste apresentam menores oscilações ao longo do ano.

Por meio de estimativas, pela aplicação da equação de Hailwood \& Harrobin de um hidrato, Mendes \& Arce (2003) obtiveram valor médio anual de UEM de 15,12\%, com mínimo de 13,61\% (setembro) e máximo de 16,33\% (abril), e pela equação de Hailwood \& Harrobin de dois hidratos, média anual de $15,00 \%$, com mínimo e máximo de $13,51 \%$ e 16,20\%, respectivamente. Galvão (1975) verificou valores médios de UEM, para diferentes cidades brasileiras, variando de $12,2 \%$ (Brasília) a 18,8\%

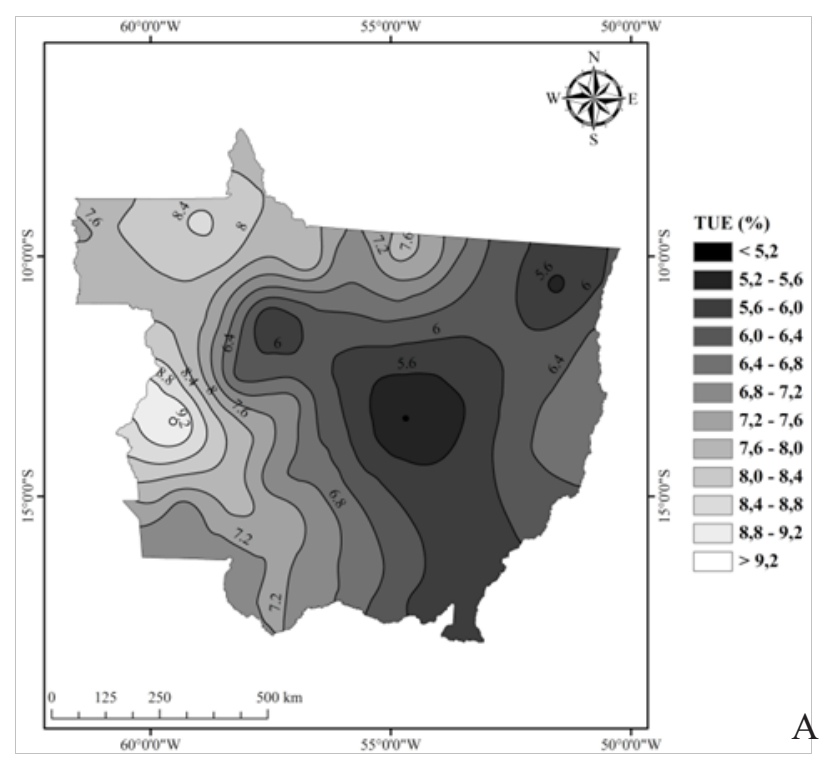

(Belém). Segundo Martins et al. (2003), pela aplicação do modelo de Simpson (1971), na região Norte e parte da região litorânea brasileira, as médias anuais de UEM variam de 16 a $18 \%$, enquanto para o estado do Mato Grosso, as oscilações ocorrem entre 14 e $16 \%$.

Os resultados obtidos para todos os municípios avaliados são inferiores aos valores observados na literatura, inclusive para a região Amazônica do Estado (com maiores níveis de UR e precipitações pluviais). Cassiano et al. (2013) observaram para a cidade de Sinop, MT, que as menores variações sazonais do UEM foram obtidas por estimativas com o modelo de Hailwood \& Harrobin para dois hidratos, com médias mensais oscilando entre 10,87 e $12,45 \%$ em setembro e janeiro, respectivamente. Ainda nessa mesma cidade, os autores verificaram que a média anual do UEM, estimada pelo método de Simpson (1971), foi de 10,68 $\pm 4,15 \%$. A distribuição espacial dos valores médios de UEM para o estado de Mato Grosso demonstram variações médias de UEM de 5,08 a $9,57 \%$ e de 8,58 e $13,49 \%$ para as estações seca e chuvosa (Figura 2), respectivamente, e de 8,3 a $12,1 \%$ na média anual (Tabela 5 e Figura 3).

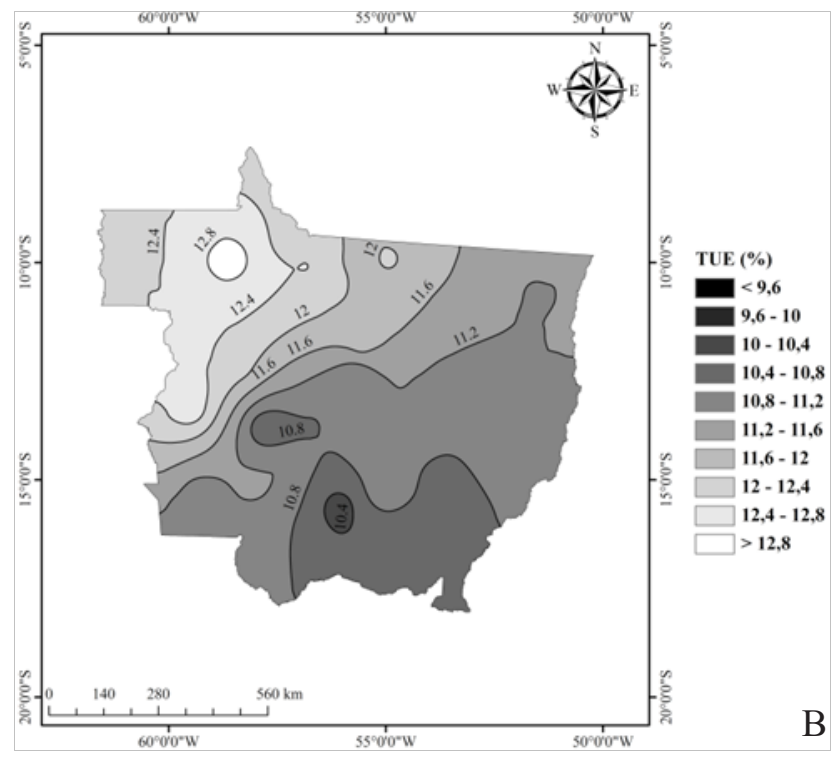

Figura 2. Distribuição especial dos valores médios da umidade de equilíbrio da madeira (em \%) nas estações seca (A) e chuvosa (B) no Estado de Mato Grosso. 


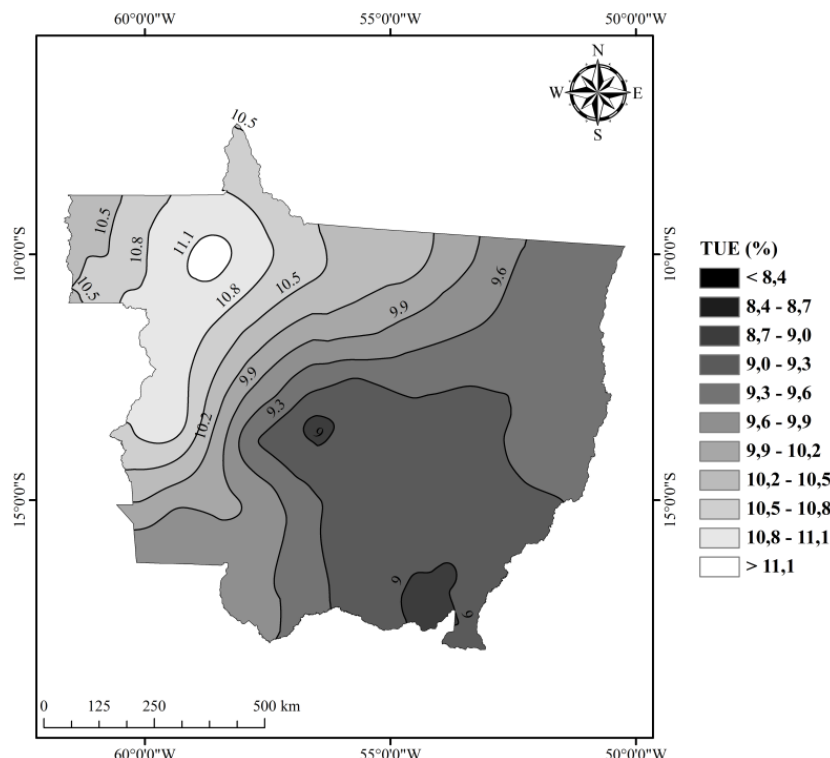

Figura 3. Distribuição espacial média anual da umidade de equilíbrio da madeira (em \%) para o Estado de Mato Grosso. Tabela 5. Valores anuais de teor de umidade de equilíbrio (em \%) para as diferentes regiões de planejamento no estado de Mato Grosso.

\begin{tabular}{lccccc}
\hline \multicolumn{1}{c}{ Regiões } & $\begin{array}{c}\mathbf{N}^{\circ} \text { da } \\
\text { região }\end{array}$ & Máximo & Mínimo & Média & Amplitude \\
\hline Noroeste 1 & I & 15,21 & 5,67 & 11,00 & 9,54 \\
Norte & II & 13,57 & 4,76 & 10,00 & 8,81 \\
Nordeste & III & 12,61 & 3,60 & 8,84 & 9,01 \\
Leste & IV & 12,55 & 3,80 & 8,81 & 8,75 \\
Sudeste & V & 13,02 & 4,11 & 8,85 & 8,91 \\
Sul & VI & 11,19 & 4,24 & 8,67 & 6,95 \\
Sudoeste & VII & 14,36 & 5,55 & 10,38 & 8,81 \\
Oeste & VIII & 14,29 & 3,99 & 9,15 & 10,30 \\
Centro Oeste & IX & 12,49 & 4,27 & 8,32 & 8,22 \\
Centro & X & 14,01 & 3,91 & 8,56 & 10,10 \\
Noroeste 2 & XI & 12,47 & 3,56 & 8,25 & 8,91 \\
Centro Norte & XII & 13,85 & 4,60 & 9,92 & 9,25 \\
\hline
\end{tabular}

Por conseguinte, as condições ambientais entre julho e setembro favorecem a secagem natural (ao ar livre) da madeira serrada, por condicionarem uma secagem mais rápida e um teor de umidade final baixo. Segundo Jankowsky et al. (1986), municípios que apresentam UE superior a $15 \%$ influenciam negativamente no processo de secagem natural (ao ar livre) da madeira, uma vez que a madeira dificilmente atingirá UEM inferior a esse valor. Sendo assim, os produtos manufaturados com essa madeira apresentarão sérios problemas de retratibilidade, se forem comercializados em regiões de clima mais seco. De acordo com Cassiano et al. (2013), os meses que apresentaram os menores valores da UEM de espécies amazônicas expostas em ensaios de campo na cidade de Sinop, região Norte de Mato Grosso, foram julho, agosto e setembro (estação seca), sendo que nesses meses, ocorreram variações de UEM entre 6,00 e $8,50 \%$, valores similares aos estimados pelo modelo de Simpson (1971), na mesma estação hídrica, para as diferentes cidades do Mato Grosso estudadas no presente trabalho.

As maiores médias anuais de UEM ocorreram nas áreas amazônicas do estado de Mato Grosso (Tabela 5), principalmente nas regiões Noroeste 1 , Norte e Centro Norte, com valores entre 9,92 e 11,00\%.

De modo geral, o estado de Mato Grosso apresenta boas condições climáticas para secagem natural da madeira, uma vez que a UEM estimada para as diferentes regiões do estado enquadram-se dentro do percentual de umidade final requerido para a madeira em diferentes usos de maior agregação de valor, como por exemplo, pisos, lambris, móveis e madeira para construção interna em geral. Contudo, ressalta-se que apenas a obtenção de uma baixa umidade de equilíbrio não é garantia de obtenção de madeira de qualidade, visto que a ausência do correto monitoramento do teor de umidade da madeira, juntamente com os erros verificados no empilhamento e/ou no pátio de secagem são fatores que podem comprometer a qualidade do produto final (Anjos et al., 2011).

\section{Conclusão}

O Estado de Mato Grosso apresenta grande amplitude na variação da umidade de equilíbrio da madeira, com maiores médias anuais na região Amazônica, quando comparado com Cerrado e Pantanal.

A umidade de equilíbrio da madeira apresenta maiores e menores médias mensais nas estações chuvosa (10,4 a $12,8 \%$ ) e seca $(5,6$ a $9,2 \%)$, independente da região do estado.

A variação sazonal da umidade de equilíbrio observada para todo o estado do Mato Grosso propiciará maior aproveitamento dos processos de secagem ao ar livre e entendimento dos comportamentos dimensionais da madeira em uso. 


\section{Referências}

Alves, E. D. L. \& Biudes, M. S. Padrões da temperatura do ar e da umidade relativa: estudo de caso no campus de Cuiabá da Universidade Federal de Mato Grosso. Boletim de Geografia, v. 30, n. 3, p. 5-16, 2012. DOI: 10.4025/bolgeogr.v30i3.13114.

Anjos, V. A. et al. Caracterização do processo de secagem da madeira nas serrarias do município de Sinop, Mato Grosso. Ciência da Madeira, v. 2, n. 1, p. 53-63, 2011. DOI: 10.12953/2177-6830. v02n01a05.

Associação Brasileira de Normas Técnicas. NBR 7190: projeto de estruturas de madeira. Rio de Janeiro, 1997. 107 p.

Baraúna, E. E. P. \& Oliveira, V. S. Umidade de equilíbrio da madeira de Angelim vermelho (Dinizia excelsa Ducke), guariúba (Clarisia racemosa Ruiz \& Pav.) e taurarí vermelho (Cariniana micrantha Ducke) em diferentes condições de temperatura e umidade relativa. Acta Amazonica, v. 39, n. 1, p. 91-96, 2009. DOI: 10.1590/S004459672009000100009.

Baronasa, R. et al. Modelling of moisture movement in wood during outdoor storage. Nonlinear analysis: modeling and control, v. 6, n. 2, p. 3-14, 2001.

Cassiano, C. et al. Sazonalidade e estimativas da umidade de equilíbrio de madeiras amazônicas em Sinop, Estado do Mato Grosso. Scientia Forestalis, v. 41, n. 100, p. 457-468, 2013.

Chen, Z. et al. Equilibrium moisture content of Norway spruce at low temperature. Wood and Fiber Science, v. 41, n. 3, p. 325-328, 2009.

Fioresi, T. et al. Umidade de equilíbrio da madeira na região Norte do Rio Grande do Sul em diferentes estações do ano. Ciência da Madeira, v. 5, n. 1, p. 34-41, 2014.

Galvão, A. P. M. Estimativas da umidade de equilíbrio da madeira em diferentes cidades do Brasil. IPEF, v. 11, n. 1, p. 53-65, 1975.

Hailwood, A. J. \& Harrobin, S. Absorption of water by polymers: analysis in terms of a simple model. Transactions of the Faraday Society, v. 42b, p. 84-102, 1946.

Jankowsky, I. P. et al. Estimativas da umidade de equilíbrio para cidades da região Sul do Brasil. IPEF, v. 32, n. 1, p. 61-64, 1986.

Jankowsky, I. P. \& Galvão, A. P. M. Influência do teor de extrativos na umidade de equilíbrio da madeira. IPEF, v. 8, n. 1, p. 1-33, 1979.

Kilic, A. \& Niemz, P. Extractives in some tropical woods. European Journal of Wood and Wood Products, v. 70, n. 1, p. 79-83, 2012. DOI: $10.1007 / \mathrm{s} 00107-010-0489-8$

Kollmann, F. F. P. \& Côte Junior, W. A. Principles of wood science and technology. New York: Springer - Verlag, 1968. 592 p.
Martins, V. A. et al. Umidade de equilíbrio e risco de apodrecimento da madeira em condições de serviço no Brasil. Brasil Florestal, v. 22, n. 76, p. 29-34, 2003.

Mato Grosso. Secretaria de Estado de Meio Ambiente do Mato Grosso. Mapa síntese do zoneamento socioeconômico ecológico - ZSEE. 2008. Disponível em: <http://www.seplan.mt.gov.br//3704951-zsee?ciclo=cv_gestao_inf $>$. Acesso em: 30 jun. 2014.

Medeiros, R. M. et al. Variabilidade da umidade relativa do ar e da temperatura máxima na bacia hidrográfica do Rio Uruçuí Preto.

Revista Educação Agrícola Superior, v. 28, n. 1, p. 44-50, 2013. DOI: 10.12722/0101-756X.v28n01a07.

Mendes, L. M. \& Arce, J. E. Análise comparativa das equações utilizadas para estimar a umidade de equilíbrio da madeira. Cerne, v. 9, n. 2, p. 141-152, 2003.

Panshin, A. J. \& De Zeeuw, C. Textbook of wood technology. 4 ed. New York: McGraw-Hill, 1980. 722 p.

Pérez-Peña, N. et al. Predicción del contenido de humedad de equilibrio de la madera em función del peso específico de la pared celular y variables ambientales. Maderas. Ciencia y Tecnología, v. 13, n. 3, p. 253-266, 2011. DOI: 10.4067/S0718221X2011000300002.

Queiroz, A. T. \& Costa, R. A. Caracterização e variabilidade climática em séries de temperatura, umidade relativa do ar e precipitação em Ituiutaba - MG. Caminhos de Geografia, v. 13, n. 43, p. 346-357, 2012.

Santos, R. B. et al. Planejamento da pulverização de fungicidas em função das variáveis meteorológicas na região de Sinop - MT. Global Science and Technology, v. 6, n. 1, p. 72-88, 2013. DOI: 10.14688/1984-3801.v06n01a07

Silva, J. C. \& Oliveira, J. T. S. Avaliação das propriedades higroscópicas da madeira de Eucalyptus saligna Sm., em diferentes condições de umidade relativa do ar. Revista Árvore, v. 27, n. 2, p. 233-239, 2003.

Simpson, W. T. Equilibrium moisture content prediction for wood. Forest Products Journal, v. 21, n. 5, p. 48-49, 1971.

Skaar, C. Water in wood. New York: Syracuse University Press, 1972. 218p.

Souza, A. P. et al. Classificação climática e balanço hídrico climatológico no estado de Mato Grosso. Nativa, v. 1, n. 1, p. 34-43, 2013. DOI: $10.1453 / 2318-7670 . v 01 n 01 \mathrm{a} 07$.

Streck, N. A. et al. Variabilidade interdecadal na série secular de temperatura do ar em Santa Maria, RS. Pesquisa Agropecuária Brasileira, v. 46, n. 8, p. 781-790, 2011. DOI: 10.1590/S0100204X2011000800001.

Varejão-Silva, M. A. Meteorologia e climatologia. Brasília, DF: INMET, 2006. 463 p. 
